\title{
HPV vaccination: triumphs and tribulations
}

\author{
Richard Hurley features and debates editor
}

The BMJ

Two studies this week concern strategies to reduce cervical cancers: by screening and by human papillomavirus vaccination. A case-control study from Sweden finds that women who attended cervical cancer screening at recommended intervals have a fifth the risk of adenosquamous cell carcinoma and a third the risk of rare types of invasive cervical carcinoma as women who did not attend screening as recommended (doi:10. 1136/bmj.11207). Jiayao Lei and colleagues explain that although the risk reductions are statistically significant the small number of cases reduces precision and precludes adjustment for lifestyle factors.

And a Scottish study concludes that introducing routine vaccination of girls aged 12 or 13 with bivalent HPV vaccine reduced high grade preinvasive cervical disease (doi:10.1136/ bmj.11161). Tim Palmer and colleagues, who analysed anonymised data from cervical screening of nearly 140000 women, say that their findings also indicate evidence of herd immunity. The data were from the first screening visit, however, and vaccinated women were over-represented, which might exaggerate the benefits of vaccination.

HPV also causes cancers in men, although these are rarer than cervical cancer. Currently, in the UK only girls and women are routinely offered vaccination. Since last year in England, men younger than 45 who have sex with men have been eligible for HPV vaccination.

Last July the Joint Committee on Vaccination and Immunisation, the UK government's independent advisory body, recommended, on scientific and economic grounds, that all boys as well as girls should be vaccinated (doi:10.1136/bmj.k3163). Routine vaccination of boys aged 12 or 13 is expected to start soon in England, but the government has ruled out a catch-up programme for older boys (http://bit.ly/2FOW0NB).

In the US the Centers for Disease Control and Prevention recommends that the HPV vaccine should be available to all schoolchildren. Last month the journalist Mara Kardas-Nelson discussed concerns about uptake of HPV vaccination among boys and men in the US (doi:10.1136/bmj.11210). The problem, she found, may be that the vaccine "is primarily seen as a preventive tool against cervical cancer" and that male vaccination is erroneously viewed by men as simply a means to protect female partners. Meanwhile, the incidence of anal cancers caused by HPV is rising in the US, and HPV has overtaken tobacco smoking as the primary cause of oropharyngeal cancers. These cancers affect men more than women and might outpace cervical cancer in a few years.

The triumphs and tribulations of HPV vaccination are, it seems, set to continue. 